\title{
Kuantum Kodlar
}

\section{Abdullah DERTLI ${ }^{1 *}$, Yasemin ÇENGELLENMIŞฺ ${ }^{1}$}

ÖZET: $\mathrm{Bu}$ çalışmada, $u^{2}=1, v^{3}=v, u v=v u, q=p^{m} \quad$ ve $\quad p \quad$ tek asal sayı olmak üzere $R=F_{q}+u F_{q}+v F_{q}+u v F_{q}+v^{2} F_{q}+u v^{2} F_{q}$ halkası üzerindeki devirli kodlar kullanılarak $F_{q}$ üzerindeki kuantum kodlar elde edildi. Ayrıca $F_{q}$ üzerindeki kuantum kodların parametreleri belirlendi. Bazı örnekler verildi.

Anahtar kelimeler: Kuantum kodlar, devirli kodlar, Gray dönüşümü.

\section{Quantum Codes}

ABSTRACT: In the present paper, the quantum codes over $F_{q}$ which are obtained from cyclic codes over the finite ring $R=F_{q}+u F_{q}+v F_{q}+u v F_{q}+v^{2} F_{q}+u v^{2} F_{q}$ with $u^{2}=1, v^{3}=v, u v=v u$, where $q=p^{m}$ and $p$ is an odd prime. Moreover the parameters of quantum codes over $F_{q}$ are determined. Some examples are given.

Keywords: Quantum codes, cyclic codes, Gray map.

\footnotetext{
${ }^{1}$ Abdullah DERTLİ (Orcid ID: 0000-0001-8687-032X), Ondokuz Mayıs Üniversitesi, Fen Edebiyat Fakültesi, Matematik Bölümü, Samsun, Türkiye

${ }^{2}$ Yasemin ÇENGELLENMIŞ (Orcid ID: 0000-0002-8133-9836), Trakya Üniversitesi, Fen Fakültesi, Matematik Bölümü, Edirne, Türkiye

*Sorumlu Yazar/Corresponding Author: Abdullah DERTLİ, e-mail: abdullah.dertli@gmail.com
} 


\section{GíRiş}

Kuantum hesaplama ve kuantum iletişimde kullanılan kuantum kodlar, kuantum bilginin taşınması sırasında oluşabilecek hataların tespiti ve düzeltilmesi için kullanılır.

Kuantum hata düzeltici kodlar ilk olarak Shor ve Steane tarafindan birbirinden bağımsız olarak elde edilmiştir (Shor, 1995; Steane, 1996).

Klasik kodlama teorisi, kuantum teorisinden farklı olmasına rağmen Calderbank ve ark. bu iki teori arasındaki geçişi bulmuşlardır (Calderbank ve ark., 1998). Bu makalede sonlu cisimler üzerindeki kodlardan kuantum kodlar elde edilmiştir.

J. Qian ve ark. $F_{2}+u F_{2}, u^{2}=0$ sonlu halkası üzerindeki devirli kodlardan kuantum hata düzeltici kodları elde etmişlerdir (Qian ve ark., 2009). X. Kai, S. Zhu, $F_{4}+u F_{4}, u^{2}=0$ halkası üzerinde (Kai ve Zhu, 2011), X. Yin ve W. Ma, $F_{2}+u F_{2}+u^{2} F_{2}, u^{3}=0$ halkası üzerinde (Yin ve Ma, 2011), devirli kodları kullanarak kuantum kodların parametrelerini oluşturmuşlardır. J. Qian, $F_{2}+v F_{2}, v^{2}=v$ halkası üzerinde keyfi uzunlukta devirli kodlardan kuantum kodları bulmuştur (Qian, 2013). (Ashraf ve Mohammed, 2014; Ashraf ve Mohammed, 2015; Ashraf ve Mohammed, 2016), M. Ashraf ve G. Mohammed $F_{3}+v F_{3}, v^{2}=1, F_{p}+v F_{p}, v^{2}=v$ ve $F_{q}+u F_{q}+v F_{q}+u v F_{q}, u^{2}=u, v^{2}=v, u v=v u \quad$ halkalar üzerinde devirli kodlardan kuantum kodların parametrelerini elde etmişlerdir. A. Dertli ve ark. sonlu halkaları kullanarak kuantum kodların parametrelerini devirli kodlardan elde etmişlerdir (Dertli ve ark., 2015a; Dertli ve ark., 2015b; Dertli ve ark., 2015c; Dertli ve ark., 2016b). Ayrıca A. Dertli ve ark. $R_{3}, R_{p}$ halkalarını kullarak negacyclic kodlardan kuantum kodları oluşturmuşlardır (Dertli ve ark., 2015d; Dertli ve ark., 2016a).

Liu Yan ve ark. $u^{2}=1, v^{3}=v, u v=v u, q=p^{m}, \quad p \quad$ tek asal say1 olmak üzere $R=F_{q}+u F_{q}+v F_{q}+u v F_{q}+v^{2} F_{q}+u v^{2} F_{q}$ halkası üzerinde skew cyclic kodları çalışmışlardır (Liu ve ark., 2017). Biz ise bu çalışmada Liu Yan ve ark. makalesindeki $R$ sonlu halkasını kullanarak devirli kodlardan kuantum kodlar elde ettik. Çalışmamızın 2. bölümünde halka ile ilgili temel bilgiler verildi. 3. bölümünde $R$ halkası üzerindeki devirli kodların dualini içermesi için gerekli ve yeterli şart verildi. Son olarak bazı örnekler elde edildi.

\section{MATERYAL VE YÖNTEM}

İlk olarak Liu Yan ve ark.

$$
\begin{aligned}
R & =F_{q}+u F_{q}+v F_{q}+u v F_{q}+v^{2} F_{q}+u v^{2} F_{q} \\
& =\left\{a_{1}+u a_{2}+v a_{3}+u v a_{4}+v^{2} a_{5}+u v^{2} a_{6}: a_{j} \in F_{q}, 1 \leq j \leq 6\right\}
\end{aligned}
$$

sonlu halkasını incelediler. Ayrıca bu çalışmalarında $R_{1}=F_{q}+u F_{q}, u^{2}=1$ halkasını kullanarak $F_{q}[u, v] /<u^{2}-1, v^{3}-v, u v-v u>$ halkasını $R=R_{1}+v R_{1}+v^{2} R_{1}$ olarak gösterdiler (Liu ve ark., 2017).

Liu Yan ve ark. $a=a_{1}+u a_{2}+v a_{3}+u v a_{4}+v^{2} a_{5}+u v^{2} a_{6}, a_{j} \in F_{q}, j=1,2, \ldots, 6, a, b, c \in R_{1}, s, t \in F_{q}$ olmak üzere

$$
\begin{gathered}
\varphi: R \rightarrow R_{1}^{3} \\
a+v b+v^{2} c \mapsto(a, a+b+c, a-b+c)
\end{gathered}
$$




$$
\begin{aligned}
\phi_{1} & : R_{1} \rightarrow F_{q}^{2} \\
s+u t & \mapsto(s, t)
\end{aligned}
$$

Gray dönüşümlerini kullanarak aşağıdaki $\Phi$ Gray dönüşümünü tanımladılar.

$$
\begin{aligned}
\Phi: R \rightarrow F_{q}^{6} \\
a \mapsto\left(a_{1}, a_{2}, a_{1}+a_{3}+a_{5}, a_{2}+a_{4}+a_{6}, a_{1}-a_{3}+a_{5}, a_{2}-a_{4}+a_{6}\right)
\end{aligned}
$$

$F_{q}$ üzerinde $x$ ve $y$ vektörlerinin $d_{H}(x, y)$ Hamming uzaklığ $1 \quad x-y$ vektörünün Hamming ağırlığına eşittir. $x=\left(x_{0}, \ldots, x_{n-1}\right) \in R^{n}$ elemanının Lee ağırlığ $1 w_{L}(x), w_{L}(x)=w_{H}(\Phi(x))$, herhangi bir $x, y \in R^{n}$ elemanlarının Lee uzaklığı $d_{L}(x, y), d_{L}(x, y)=w_{L}(x-y)$ olarak tanımlanır.

Teorem 1: Gray dönüşümü $\Phi$ uzaklık koruyan bir dönüşümdür ve $F_{q}$-lineerdir (Liu ve ark., 2017).

$R$ üzerinde $n$ uzunluğa sahip $\operatorname{bir} C$ lineer kodu $R^{n} \operatorname{nin} \operatorname{bir} R$-altmodülüdür.

Lemma 2: $C, R$ üzerinde $n$ uzunluğa sahip, rankı $k$ ve minimum Lee uzaklığı $d$ olan bir lineer kod olsun. Bu durumda $\Phi(C), F_{q}$ üzerinde bir $[6 n, k, d]$ lineer bir koddur (Liu ve ark., 2017).

Herhangi bir $x=\left(x_{0}, \ldots, x_{n-1}\right), y=\left(y_{0}, \ldots, y_{n-1}\right)$ elemanlarının iç çarpımı aşağıdaki gibi tanımlanır

$$
x y=\sum_{i=0}^{n-1} x_{i} y_{i}
$$

Eğer $x y=0$ ise $x$ ve $y$ elemanları ortogonaldir denir. $C, R$ üzerinde $n$ uzunluğa sahip bir lineer kod olmak üzere $C$ kodunun duali

$$
C^{\perp}=\{x: \forall y \in C, x y=0\}
$$

şeklindedir. $C$ kodunun duali de $R$ üzerinde $n$ uzunluğa sahip lineer bir koddur. Eğer $C \subset C^{\perp}$ ise $C$ koduna self ortogonal kod, $C=C^{\perp}$ ise $C$ koduna self dual kod denir.

Teorem 3: $C, R$ üzerinde $n$ uzunluğa sahip lineer bir kod olsun. Bu durumda $\Phi(C)^{\perp}=\Phi\left(C^{\perp}\right)$. Ayrica, $C$ self dual kod ise $\Phi(C)$ kodu da self dual koddur (Liu ve ark., 2017).

(Liu ve ark., 2017), Çin Kalan Teoremini kullanarak $R$ halkasını aşağıdaki şekilde ifade etmiştir.

$$
\begin{gathered}
R=2^{-1}(1+u)\left(1-v^{2}\right) F_{q} \oplus 2^{-1}(1-u)\left(1-v^{2}\right) F_{q} \oplus 4^{-1}(1+u)\left(v+v^{2}\right) F_{q} \\
\oplus 4^{-1}(1-u)\left(v+v^{2}\right) F_{q} \oplus 4^{-1}(1+u)\left(-v+v^{2}\right) F_{q} \oplus 4^{-1}(1-u)\left(-v+v^{2}\right) F_{q} \\
\eta_{1}=2^{-1}(1+u)\left(1-v^{2}\right), \eta_{2}=2^{-1}(1-u)\left(1-v^{2}\right) \\
\eta_{3}=4^{-1}(1+u)\left(v+v^{2}\right), \eta_{4}=4^{-1}(1-u)\left(v+v^{2}\right) \\
\eta_{5}=4^{-1}(1+u)\left(-v+v^{2}\right), \eta_{6}=4^{-1}(1-u)\left(-v+v^{2}\right)
\end{gathered}
$$

olmak üzere $R$ üzerinde $n$ uzunluğa sahip $C$ lineer kodu

$$
C=\sum_{j=1}^{6} \eta_{j} C_{j}
$$


şeklinde ifade edilir. Burada ki $C_{j}=\left\{x_{j} \in F_{q}^{n}: \exists x_{i} \in F_{q}^{n}, i \in\{1,2, \ldots, 6\} \backslash\{j\}, \sum_{i=1}^{6} \eta_{i} x_{i} \in C\right\}$ kodları $F_{q}$ üzerinde $n$ uzunluğa sahip lineer kodlardır (Liu ve ark., 2017).

$G_{1}, G_{2}, \ldots, G_{6}$, sırasıyla $C_{1}, C_{2}, \ldots, C_{6}$ kodlarının üreteç matrisleri ise $C$ kodunun üreteç matrisi

$$
G=\left[\begin{array}{c}
\eta_{1} G_{1} \\
\eta_{2} G_{2} \\
\vdots \\
\eta_{6} G_{6}
\end{array}\right]
$$

dir. $d_{L}, C$ kodunun minimum Lee ağırlığ 1 olsun. $d_{H}\left(C_{i}\right)$, sirasıyla $C_{1}, C_{2}, \ldots, C_{6}$ kodlarının minimum Hamming ağırlı̆̆ olmak üzere,

$$
d_{L}=d_{H}(\Phi(C))=\min \left\{d_{H}\left(C_{1}\right), d_{H}\left(C_{2}\right), \ldots, d_{H}\left(C_{6}\right)\right\}
$$

dir (Liu ve ark., 2017).

Önerme 4: $i=1,2, \ldots, 6$ için $C_{i}, \quad F_{q}$ üzerinde $n$ uzunluğa sahip lineer kodlar olmak üzere $C=\eta_{1} C_{1} \oplus \eta_{2} C_{2} \oplus \eta_{3} C_{3} \oplus \eta_{4} C_{4} \oplus \eta_{5} C_{5} \oplus \eta_{6} C_{6}, R$ üzerinde $n$ uzunluğa sahip lineer bir kod olsun. $C$ kodunun devirli bir kod olması için gerekli ve yeterli koşul $i=1,2, \ldots, 6$ için $C_{i}$ kodlarının devirli kod olmasidir.

İspat: $C$ devirli bir kod, $\left(a_{0}^{1}, a_{1}^{1}, \ldots, a_{n-1}^{1}\right) \in C_{1},\left(a_{0}^{2}, a_{1}^{2}, \ldots, a_{n-1}^{2}\right) \in C_{2}, \ldots,\left(a_{0}^{6}, a_{1}^{6}, \ldots, a_{n-1}^{6}\right) \in C_{6}$ olmak üzere $i=0,1, \ldots, n-1 \quad$ için $m_{i}=\eta_{1} a_{i}^{1}+\eta_{2} a_{i}^{2}+\ldots+\eta_{6} a_{i}^{6} \quad$ olsun. $C$ kodu devirli bir kod olduğundan $\left(m_{0}, m_{1}, \ldots, m_{n-1}\right) \in C \operatorname{için}\left(m_{n-1}, m_{0}, \ldots, m_{n-2}\right) \in C$ dir. Bu durumda,

$$
\left(m_{n-1}, m_{0}, \ldots, m_{n-2}\right)=\eta_{1}\left(a_{n-1}^{1}, a_{0}^{1}, \ldots, a_{n-2}^{1}\right)+\eta_{2}\left(a_{n-1}^{2}, a_{0}^{2}, \ldots, a_{n-2}^{2}\right)+\ldots+\eta_{6}\left(a_{n-1}^{6}, a_{0}^{6}, \ldots, a_{n-2}^{6}\right)
$$

eşitliği elde edilir. Böylece $\left(a_{n-1}^{1}, a_{0}^{1}, \ldots, a_{n-2}^{1}\right) \in C_{1}, \ldots,\left(a_{n-1}^{6}, a_{0}^{6}, \ldots, a_{n-2}^{6}\right) \in C_{6}$. O halde $C_{1}, C_{2}, \ldots, C_{6}$ kodları devirli koddur.

Tersine, $C_{1}, C_{2}, \ldots, C_{6}$ kodları devirli kod olsun. $\mathrm{Bu}$ durumda $i=0,1, \ldots, n-1$ için $m_{i}=\eta_{1} a_{i}^{1}+\eta_{2} a_{i}^{2}+\ldots+\eta_{6} a_{i}^{6}$ olmak $\quad$ üzere $\quad\left(m_{0}, m_{1}, \ldots, m_{n-1}\right) \in C \quad$ ve $\quad\left(a_{0}^{1}, a_{1}^{1}, \ldots, a_{n-1}^{1}\right) \in C_{1}, \ldots$, $\left(a_{0}^{6}, a_{1}^{6}, \ldots, a_{n-1}^{6}\right) \in C_{6}$ olur. Buradan

$$
\left(m_{n-1}, m_{0}, \ldots, m_{n-2}\right)=\eta_{1}\left(a_{n-1}^{1}, a_{0}^{1}, \ldots, a_{n-2}^{1}\right)+\eta_{2}\left(a_{n-1}^{2}, a_{0}^{2}, \ldots, a_{n-2}^{2}\right)+\ldots+\eta_{6}\left(a_{n-1}^{6}, a_{0}^{6}, \ldots, a_{n-2}^{6}\right) \in C
$$

elde edilir. O halde $C$ devirli bir koddur.

Önerme 5: $C=\sum_{j=1}^{6} \eta_{j} C_{j}, R$ üzerinde $n$ uzunluğa sahip devirli bir kod olsun. O zaman $f_{1}, f_{2}, \ldots, f_{6}$ sırasıyla $C_{1}, C_{2}, \ldots, C_{6}$ kodlarının üreteç polinoları olmak üzere

$$
C=\left\langle\eta_{1} f_{1}, \eta_{2} f_{2}, \ldots, \eta_{6} f_{6}\right\rangle
$$

dir. 
Lemma 6: $C=\sum_{j=1}^{6} \eta_{j} C_{j}, R$ üzerinde $n$ uzunluğa sahip devirli bir kod olsun. Bu durumda $f_{1}, f_{2}, \ldots, f_{6}$ sırasıyla $C_{1}, C_{2}, \ldots, C_{6}$ kodlarının üreteç polinoları ve

$$
f(x)=\eta_{1} f_{1}(x)+\eta_{2} f_{2}(x)+\ldots+\eta_{6} f_{6}(x)
$$

olmak üzere $C=\langle f(x)\rangle$ olacak şekilde tek bir $f(x)$ polinomu vardır ve $f(x) \mid x^{n}-1$.

Lemma 7: $C=\sum_{j=1}^{6} \eta_{j} C_{j}, R$ üzerinde $n$ uzunluğa sahip devirli bir kod olsun. Bu durumda $i=1,2, \ldots, 6$ için $h_{i}^{*}(x)=x^{\operatorname{deg} h_{i}(x)} h_{i}\left(x^{-1}\right), h_{i}(x)=\left(x^{n}-1\right) / f_{i}(x)$, reciprocal polinomları olmak üzere

$$
C^{\perp}=\left\langle\eta_{1} h_{1}^{*}+\eta_{2} h_{2}^{*}+\ldots+\eta_{6} h_{6}^{*}\right\rangle
$$

eşitliği elde edilir.

Lemma 8: $f^{*}(x), f(x)$ polinomunun reciprocal polinomu olmak üzere $f(x)$ polinomu ile üretilen devirli bir $C$ kodunun dualini içermesi için gerekli ve yeterli koşul

$$
x^{n}-1 \equiv 0\left(\bmod f f^{*}\right)
$$

olmasidır (Ashraf ve Mohammed, 2017).

\section{BULGULAR VE TARTIŞMA}

Lemma 9: $C_{1}$ ve $C_{2}, F_{q}$ üzerinde tanımlı sirasıyla $\left[n, k_{1}, d_{1}\right]_{q}$ ve $\left[n, k_{2}, d_{2}\right]_{q}$ parametrelerine sahip lineer $\operatorname{kod}, C_{2}^{\perp} \subseteq C_{1}$,

$$
d=\min \left\{w_{t}(v): v \in\left(C_{1} \backslash C_{2}^{\perp}\right) \cup\left(C_{2} \backslash C_{1}^{\perp}\right)\right\} \geq \min \left\{d_{1}, d_{2}\right\}
$$

olsun. Bu durumda $\left[\left[n, k_{1}+k_{2}-n, d\right]_{q}\right.$ parametrelerine sahip bir $C$ kuantum hata düzeltici kod vardır (Ashraf ve Mohammed, 2017).

Teorem 10: $f(x)=\eta_{1} f_{1}(x)+\eta_{2} f_{2}(x)+\ldots+\eta_{6} f_{6}(x)$ olmak üzere $C=\langle f(x)\rangle, R$ üzerinde keyfi $n$ uzunluğa sahip devirli bir kod olsun. Bu durumda $C^{\perp} \subseteq C$ olması için gerekli ve yeterli koşul $i=1, \ldots, 6$ için $f^{*}(x), f(x)$ polinomunun reciprocal polinomu olmak üzere $x^{n}-1 \equiv 0\left(\bmod f_{i}(x) f_{i}^{*}(x)\right)$ olmasıdır.

İspat: $x^{n}-1 \equiv 0\left(\bmod f_{i}(x) f_{i}^{*}(x)\right)$ olsun. Lemma 8 den $C_{i}^{\perp} \subseteq C_{i}$ dir. Buradan $\eta_{i} C_{i}^{\perp} \subseteq \eta_{i} C_{i}$ ve $\sum_{j=1}^{6} \eta_{j} C_{j}^{\perp} \subseteq \sum_{j=1}^{6} \eta_{j} C_{j}$ elde edilir. Böylece $\left\langle\sum_{j=1}^{6} \eta_{j} h_{j}^{*}(x)\right\rangle \subseteq\left\langle\sum_{j=1}^{6} \eta_{j} f_{j}(x)\right\rangle$. Yani, $C^{\perp} \subseteq C$ dir.

Tersine, $C^{\perp} \subseteq C$ olsun. Bu durumda $\sum_{j=1}^{6} \eta_{j} C_{j}^{\perp} \subseteq \sum_{j=1}^{6} \eta_{j} C_{j}$. $C_{i}$ koları $F_{q}$ üzerinde tanımlı olduğundan $\eta_{i} C_{i}$, modulo $\eta_{\mathrm{i}}$ ye göre $C$ koduna denktir. O halde $C_{i}^{\perp} \subseteq C_{i}$. Böylece $x^{n}-1 \equiv 0\left(\bmod f_{i}(x) f_{i}^{*}(x)\right)$ dir.

Lemmma 9 ve Teorem 10 u kullanarak kuantum kodların parametrelerini aşağıdaki gibi elde ederiz.

Teorem 11: $C=\sum_{j=1}^{6} \eta_{j} C_{j}, R$ üzerinde $n$ uzunluğa sahip devirli bir kod olsun. Eğer $i=1,2, \ldots, 6$, için $C_{i}^{\perp} \subseteq C_{i}$ ise $C^{\perp} \subseteq C$ dir ve $d_{L}, C$ kodunun minimum Lee ağırlı̆̆ı, $k, \Phi(C)$ kodunun boyutu olmak üzere $\left[\left[6 n, 2 k-6 n, d_{L}\right]\right]$ parametrelerine sahip bir kuantum hata düzeltici kod vardır. 
Örnek 1: $F_{9}=\left\{x+a y: x, y \in F_{3} a^{2}+a+2=0\right\}$ olmak üzere $x^{6}-1=(x+1)^{3}(x+2)^{3} \in F_{9}[x]$

$$
f_{1}(x)=f_{2}(x)=f_{3}(x)=f_{4}(x)=f_{5}(x)=f_{6}(x)=x+1
$$

olsun. Böylece $C=\left\langle\eta_{1} f_{1}, \eta_{2} f_{2}, \ldots, \eta_{6} f_{6}\right\rangle . C_{i}$ kodları 6 uzunluğunda $[6,5,2]$-koddur. Bu durumda $\Phi(C)$, $[36,30,2]$ parametreye sahip lineer bir koddur. Teorem 10 dan, $C^{\perp} \subseteq C$. Teorem 11 i kullanarak, $[[36,24,2]]$ parametrelerine sahip kuantum kod elde ederiz.

Örnek 2: $x^{10}-1=(x+1)^{5}(x+4)^{5} \in F_{5}[x]$

$$
f_{1}(x)=f_{2}(x)=(x+4)^{2}, f_{3}(x)=f_{4}(x)=f_{5}(x)=f_{6}(x)=(x+1)^{2}
$$

olsun. Böylece $C=\left\langle\eta_{1} f_{1}, \eta_{2} f_{2}, \ldots, \eta_{6} f_{6}\right\rangle$. $C_{i}$ kodları10uzunluğunda $[10,8,2]$-koddur. Bu durumda $\Phi(C)$ , $[60,48,2]$ parametreye sahip lineer bir koddur. Teorem 10 dan, $C^{\perp} \subseteq C$. Teorem 11 i kullanarak, $[[60,36,2]]$ parametrelerine sahip kuantum kod elde ederiz.

Çizelge 1. Kuantum Kodlar

\begin{tabular}{cccccc}
\hline$n$ & $q$ & $C_{1}=C_{2}=C_{3}$ & $C_{4}=C_{5}=C_{6}$ & $\Phi(C)$ & {$[[N, K, D]]$} \\
\hline 3 & 19 & {$[3,2,2]$} & {$[3,2,2]$} & {$[18,12,2]$} & {$[[18,6,2]]$} \\
4 & 9 & {$[4,3,2]$} & {$[4,3,2]$} & {$[24,18,2]$} & {$[[24,12,2]]$} \\
12 & 3 & {$[12,9,2]$} & {$[12,9,2]$} & {$[72,54,2]$} & {$[[72,36,2]]$} \\
20 & 9 & {$[20,16,4]$} & {$[20,16,4]$} & {$[120,96,4]$} & {$[[120,72,4]]$} \\
27 & 3 & {$[27,21,2]$} & {$[27,21,2]$} & {$[162,126,2]$} & {$[[162,90,2]]$} \\
30 & 5 & {$[30,29,2]$} & {$[30,29,2]$} & {$[180,174,2]$} & {$[[180,168,2]]$} \\
36 & 5 & {$[36,34,2]$} & {$[36,34,2]$} & {$[216,204,2]$} & {$[[216,192,2]]$} \\
\hline
\end{tabular}

\section{SONUÇ}

$\mathrm{Bu} \quad$ çalışmada, $u^{2}=1, v^{3}=v, u v=v u, q=p^{m}$ ve $p$ tek $\quad$ asal $\quad$ sayı olmak üzere $R=F_{q}+u F_{q}+v F_{q}+u v F_{q}+v^{2} F_{q}+u v^{2} F_{q}$ halkası üzerindeki devirli kodlar kullanılarak $F_{q}$ üzerindeki kuantum kodların parametreleri elde edilerek bazı sonlu cisimler üzerinde örnekler verildi. Yapılan bu çalışma farklı halkalara taşınarak yeni optimal kuantum kodlar elde edilebilir.

\section{KAYNAKLAR}

Ashraf M, Mohammad G, 2014. Quantum codes from cyclic codes over $F_{3}+v F_{3}$. International Journal of Quantum Information, 12 (6):1450042.

Ashraf M, Mohammad G, 2015. Construction of quantum codes from cyclic codes over $F_{p}+v F_{p}$. International Journal of Information and Coding Theory, 2 : 137-144.

Ashraf M, Mohammad G, Quantum codes from cyclic codes over $F_{q}+u F_{q}+v F_{q}+u v F_{q}$. Quantum Information Processing, DOI:10.1007/s11128-016-1379-8.

Calderbank A R, Rains E M, Shor P M, Sloane N J A, 1998. Quantum error correction via codes over GF(4). IEEE Transactions on Information Theory, 44:1369-1387. 
Dertli A, Cengellenmis Y, Eren S, 2015a. On quantum codes obtained from cyclic codes over $A_{2}$. International Journal of Quantum Information, 13: 1550031.

Dertli A, Cengellenmis Y, Eren S, 2015b. Quantum Codes over the Ring $F_{2}+u F_{2}+u^{2} F_{2}+\ldots+u^{m} F_{2}$. International Journal of Algebra, 9: 115-121.

Dertli A, Cengellenmis Y, Eren S, 2016a. On the linear codes over the ring $R_{p}$. Discrete Mathematics, Algorithms and Applications, 1650036.

Dertli A, Cengellenmis Y, Eren S, 2015c. On the Codes over a Semilocal Finite Ring. Intern. J. of Adv. Computer Science \& Application, DOI: 10.14569/IJACSA.2015.061038.

Dertli A, Cengellenmis Y, Eren S, 2016b. Some results on the linear codes over the finite ring $F_{2}+v_{1} F_{2}+\ldots+v_{r} F_{2}$. International Journal of Quantum Information, 1650012.

Dertli A, Cengellenmis Y, Eren S, 2015d. Quantum Codes Over $F_{2}+u F_{2}+v F_{2}$. Palestine Journal of Mathematics, 4: 547-552.

Kai X, Zhu S, 2011. Quaternary construction bof quantum codes from cyclic codes over $F_{4}+u F_{4}$. International Journal of Quantum Information, 9:689-700.

Liu Y, Shi M, Lu Z, 2017. Skew cyclic codes over $F_{q}[u, v] /<u^{2}-1, v^{3}-v, u v-v u>$. IEICE Transactions Fundamentals, DOI:101587/transfun.E0.A.1.

Qian J, 2013. Quantum codes from cyclic codes over $F_{2}+v F_{2}$. Journal of Information \& computational Science, 10:1715-1722.

Qian J, Ma W, Gou W, 2009. Quantum codes from cyclic codes over finite ring. International Journal of Quantum Information, 7:1277-1283.

Shor P W, 1995. Scheme for reducing decoherence in quantum memory. Phys. Rev. A, 52:2493-2496. Steane A M, 1996. Simple quantum error correcting codes. Phys. Rev. A, 54:4741-4751.

Yin X, Ma W, 2011. Gray map and quantum codes over the ring $F_{2}+u F_{2}+u^{2} F_{2}$. In Trust, Security and Privacy in Computing and Communications (TrustCom). IEEE 10th International Conference on, p:897-899. 